\title{
Le conflit chypriote relocalisé à Londres : pratiques transnationales de la frontière
}

Cypriot conflict relocated to London: transnational border practices

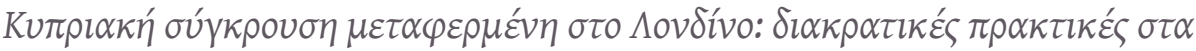

$\sigma u ́ v o \rho \alpha$

\section{Barbara Karatsioli}

\section{OpenEdition}

\section{Journals}

Édition électronique

URL : https://journals.openedition.org/ceb/14340

DOI : $10.4000 /$ ceb. 14340

ISSN : 2261-4184

Éditeur

INALCO

Édition imprimée

ISBN : 9782858313341

ISSN : 0290-7402

Référence électronique

Barbara Karatsioli, «Le conflit chypriote relocalisé à Londres : pratiques transnationales de la frontière », Cahiers balkaniques [En ligne], 46 | 2020, mis en ligne le 25 février 2020, consulté le 06 juillet 2021. URL : http://journals.openedition.org/ceb/14340 ; DOI : https://doi.org/10.4000/ceb.14340

Cahiers balkaniques est mis à disposition selon les termes de la Licence Creative Commons Attribution - Pas d'Utilisation Commerciale 4.0 International. 


\title{
Le conflit chypriote relocalisé à Londres : pratiques transnationales de la frontière
}

\author{
Cypriot conflict relocated to London: \\ transnational border practices

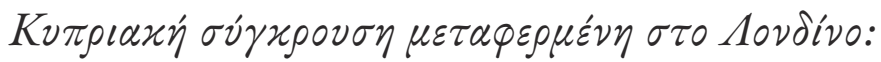

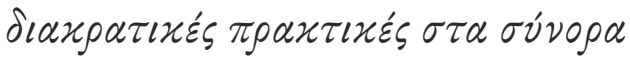

Barbara Karatsioli

Université Paris VIII

En 2007, un jeune homme d'une vingtaine d'années, né en France de père chypriote grec et de mère française, entreprend un voyage à Chypre avec un projet hors du commun: ramener la paix dans l'île divisée. Étudiant à l'université, républicain, ardent défenseur des principes issus de la Révolution française, il était convaincu que l'Europe démocratique, exempte de guerres, pouvait - notamment par son élargissement - faire souffler un vent de paix sur les pays déchirés par les conflits. En quête de ses propres origines et de celles du conflit chypriote, le jeune homme passe les deux premiers mois de ce voyage initiatique dans un couvent à Paphos, persuadé que l'essence et l'identité grecque chypriote du conflit étaient liées à la religion. C'est une fois à Nicosie, la capitale divisée, qu'il met en ouvre son plan: hisser le drapeau européen sur la cathédrale d'Aghia Sofia, devenue après la conquête ottomane de Chypre en 1571, la mosquée Selimiye. Pour ce faire, il a activé le «réseau d'amis» chypriotes turcs prêt à le soutenir qu'il avait déjà constitué sur Facebook. Hébergé dans la partie de Nicosie «sous contrôle de la République de Chypre» par la famille nationaliste de droite de son père, il a retrouvé ses amis dans la partie nord de la ville, partie occupée par l'armée turque, du checkpoint de la rue Ledra/Lokmaci. Ensemble, ils se sont dirigés vers la cathédrale d'Aghia Sofia. Là, il s'est équipé pour escalader le minaret et, faute d'avoir trouvé le drapeau du conquérant turc, il a enlevé le drapeau chypriote turc. À la place, il a érigé le drapeau de l'Union 
européenne pendant que ses amis chypriotes turcs filmaient. Sa performance achevée, il les a rejoints par l'intérieur de la mosquée. Ils ont marché ensemble jusqu'au checkpoint où ils se sont séparés. Le jeune homme a ensuite regagné la maison de sa famille chypriote grecque. Le lendemain, il a reçu la visite d'un policier chypriote grec, après quoi il s'est empressé de quitter l'île, satisfait d'avoir pu donner corps symboliquement à son idéal politique.

En hissant le drapeau européen sur l'église-mosquée, un lieu hautement symbolique, comme l'avaient fait tous les conquérants avant lui, il cherchait à modifier le sens du lieu: en faire un symbole de paix et non plus de conquête, et insuffler un nouvel imaginaire politique de tolérance et de coexistence des religions, et notamment de paix. Son geste affirmait que Chypre toute entière était un espace et un territoire européen et comme tels, devait être pacifiée. Le réseau transnational éphémère qui s'étendait vers l'ennemi de son père, qu'il a activé en vue de son spectaculaire acte politique à Chypre, devait marquer un désir conjoint pour la paix. Car cet acte performatif pour la réunification de Chypre sous le drapeau européen s'inscrit en continuité, en reconnaissance et en réponse aux mouvements massifs chypriotes turcs de 2002-2003. C'est un geste qui entérine l'approbation chypriote turque obtenue par suffrage universel lors du référendum pour la réunification de Chypre en 2004, avant l'entrée de la République de Chypre dans l'Union européenne. La Ligne verte de séparation persiste malgré l'adhésion de la République de Chypre à l'UE; elle marque désormais les limites territoriales de la souveraineté de l'UE. Au-delà, l'acquis communautaire est suspendu pour la «République turque de Chypre nord » $(\mathrm{RTCN})^{1}$, dépourvue de statut international. Nicosie ne pouvait être à la fois la capitale de la République de Chypre et celle de la RTNC. Par son geste de pacification exprimé à travers le drapeau sur l'église-mosquée, le jeune a revendiqué l'unité et la réunification de la capitale, en faisant une exigence européenne impliquant aussi la démocratisation de la partie chypriote turque.

Comme l'illustre ce cas, la volonté d'agir sur le conflit ne manque pas parmi les descendants des migrants Chypriotes. Et pourtant, la mobilisation de la ou des diasporas chypriote $(s)$ - turque et grecque - dans le conflit demeure un champ qui n'est que parcimonieusement investi, comme le constatait déjà Gilles Bertrand il y a vingt ans ${ }^{2}$. Madeline Demetriou (2003) défriche le terrain à travers l'étude des

1. La République de Chypre est indépendante en 1960. L’État, constitué alors sur une base bicommunautaire, une proto-forme du consociationalisme, est depuis 1963 et après le retrait de la communauté chypriote turque, sous contrôle des Chypriotes grecs. Le conflit s'est avéré plus durable que l'État dont on négocie la forme depuis bientôt 40 ans.

2. Bertrand, 2000. 
institutions qui gouvernent les relations de la diaspora chypriote grecque et de la République de Chypre. Le présent article propose d'y contribuer par l'analyse des activités politiques transnationales des migrants chypriotes à Londres dans le conflit chypriote. Cette approche non institutionnelle de l'activisme politique chypriote puise aux travaux d'une recherche ethnographique menée principalement entre 2001 et 2006 sur la diaspora chypriote - grecque et turque à Londres.

Évoquer un cas issu de la France en introduction de cet article lui apporte une valeur heuristique. Il pousse à souligner d'emblée que la communauté diasporique chypriote à Londres fait partie d'un espace diasporique plus étendu. Être et agir en Chypriote ou Chypriote grec et Chypriote turc sur le conflit peut acquérir un sens différent selon la localité, selon qu'on a été élevé en France ou au Royaume-Uni ${ }^{3}$. Il peut acquérir un sens différent selon que l'on fait partie de la diaspora majoritaire ( $90 \%$ ) installée dans un pays du Commonwealth britannique (Australie, Canada et Afrique du Sud), dont environ $30 \%$ se trouve concentrée au Royaume-Uni (Londres) ou que l'on appartienne aux $10 \%$ restants répartis dans le monde, dont la France ${ }^{4}$. Ceci d'autant plus que les liens historiques avec le Royaume-Uni sont récents ${ }^{5}$ : sujets britanniques jusqu'en 1960, les Chypriotes se sont implantés progressivement dans la métropole britannique et dans d'autres pays du Commonwealth à partir du début du $\mathrm{Xx}^{\mathrm{e}}$ siècle jusqu'à l'indépendance en $1960^{6}$. Être chypriote peut aussi acquérir un sens différent suivant que l'on coexiste ou pas avec l'ennemi. Le jeune français n'a pas grandi avec des Chypriotes turcs. À Londres, les Chypriotes - turcs ou grecs - vivent ou ont conscience de vivre ensemble et de perpétuer la cohabitation avec l'ennemi. Cette situation qui s'inscrit en continuité avec le passé de pré-division de Chypre ${ }^{7}$ est en radicale opposition avec la situation présente dans l'île. L'étude de la délocalisation et la

\section{Demetriou, 2003.}

4. Les chiffres approximatifs donnent quelques 500000 Chypriotes grecs en diaspora, environ 700000 Chypriotes si on compte les Chypriotes turcs. GeOrgallides, 1990 ; BERTRAND, 2000.

5. Chypre, cédée aux Britanniques en 1878 par l'Empire ottoman, devint protectorat en 1914 pour ensuite devenir colonie de la Couronne en 1924.

6. Si la diaspora londonienne est importante par rapport à l'ensemble de la diaspora chypriote du Commonwealth, nous ne cherchons nullement à l'aborder ici comme un point nodal dans l'espace transnational diasporique chypriote du Commonwealth. Ce point important est développé dans un autre travail.

7. La division de 1974 met fin à la coexistence de deux communautés du village à l'État. L'exception notable à Chypre est Pyla/Pile, village en zone tampon. 
158 Politique et sociétés à Chypre aujourd'hui

transformation du conflit chypriote dans et par la diaspora à Londres que nous proposons ici prend en compte ces trois aspects fondamentaux tout en privilégiant le dernier.

Dans un premier temps, l'article étudie le déplacement du conflit chypriote à Londres et la manière dont la frontière se recompose, intangible, sur un territoire qui n'est pas le sien, c'est-à-dire au-delà de l'île partitionnée de Chypre $^{8}$. Il considère la manière dont le conflit transforme la diaspora chypriote préexistante à Londres et affecte son tissu social (ce qui n'est pas le cas dans d'autres villes comme Paris). Dans un deuxième temps, il pointe les répercussions de ce déplacement sur la frontière physique et matérielle à Chypre au travers des actions politiques individuelles et collectives des migrants et au travers de leurs engagements politiques dans le conflit - dont la paix. Plutôt que d'aborder le rôle de la diaspora comme «promoteur » ou «briseur » de paix (“builders" or “wreckers") 9 l'article vise avant tout à comprendre les engagements transnationaux des migrants chypriotes sur la frontière et dans le conflit à Chypre et à Londres, au travers des liens qu'ils forgent à différentes échelles.

\section{Relocalisation du conflit à Londres et mutations diasporiques}

La frontière, nous l'avons dit, se recompose au-delà de l'île partitionnée de Chypre. La division de Chypre en 1974 et la relégation des Chypriotes turcs dans la partie nord et des Chypriotes grecs dans la partie sud font de Londres un des principaux sites où les ennemis cohabitent et où la frontière est renégociée au niveau des relations intercommunautaires. En ce sens, la frontière physique et matérielle de la division de l'île ne disparaît pas à Londres. Certes, elle est invisible à l'œil nu, mais l'étude de ses dynamiques fait apparaître l'évolution du conflit chez les Chypriotes déplacés et la manière dont il a aussi transformé la vie et l'identité politique de la diaspora. C'est cette mutation qu'il importe de voir ici.

La présence chypriote durable et croissante à Londres remonte aux années 1920-1930 ${ }^{10}$, quand Chypre devint une colonie de la Couronne. Les recensements officiels décennaux nous renseignent sur la constante accélération des flux migratoires et sur l'augmentation du nombre de migrants nés à

8. Cet article puise dans une enquête ethnographique sur les cohabitations entre Chypriotes grecs et turcs, dont celle de la diaspora chypriote de Londres entre 2001 et 2008; depuis lors, cette enquête se prolonge, par de fréquents allers-retours. C'est cet aspect de cohabitation des ennemis qui prime dans cette démarche.

9. Scheffer, 1993 ; Shain \& Barth, 2003 ; Smith \& Stares, 2007.

10. OACKLEY, 1979. 
Chypre, au point de devenir la troisième plus importante migration issue des pays du Commonwealth dans les années $1960^{11}$. Mais, leur inexactitude et leur inadéquation ${ }^{12}$, des années 1930 à nos jours, rendent difficile la distinction entre Chypriotes grecs et Chypriotes turcs jusqu'à la division de l'île en 1974. La combinaison de plusieurs registres - électoraux, religieux etc. -, opérée par des études démographiques dans les années 1960 et 1970, offre des informations plus précises sur les Chypriotes turcs et grecs - et parfois arméniens et roms et notamment sur leur distribution mouvante dans la ville; ces mouvements internes des communautés migrantes sont souvent étudiés dans une perspective d'intégration dans la société britannique.

Après 1974, les données concernent uniquement les Chypriotes grecs, nés dans la République de Chypre, la seule entité politique de l'île reconnue internationalement. Compte tenu de l'«illégalité » de leurs documents, les Chypriotes migrants issus de la RTCN, entité politique «illégale » n’apparaissent pas dans les recensements ${ }^{13}$. Et lorsqu' ils apparaissent dans différentes statistiques, et notamment dans des études récentes sur les communautés turcophones et sur les communautés musulmanes au Royaume-Uni ${ }^{14}$, les chiffres obtenus pour les Chypriotes turcs issus de la RTCN confondent Turcs et Kurdes.

À la nature problématique des recensements s'ajoute celle de l'analyse et de l'interprétation des données qui ramène les données statistiques aux phénomènes politiques et sociaux les plus importants de chaque décennie, donc très souvent au conflit. Enfin, l'analyse de la migration chypriote au Royaume-Uni - nous le verrons plus loin - gagnerait à être intégrée dans une analyse plus étendue du phénomène diasporique chypriote.

Un autre problème est la terminologie à employer pour décrire et analyser la présence chypriote. Nous avons utilisé le terme diaspora chypriote, sans précision quant à son emploi. Madeline Demetriou nous apprend que le mot est officiellement employé par le gouvernement de la République de Chypre après 1974, lorsqu' il amorce la diaspora organisée pour agir dans l'arène internationale ${ }^{15}$. Cet amorçage de la diaspora, une tendance adoptée par de nombreux États, inscrit les Chypriotes grecs au Royaume-Uni dans la catégorie des nouvelles communautés

11. LADBURY, 1977.

12. Bhrolchain, 1990 ; Lassalle, 2006 ; Mehmet Ali, 2001.

13. Mehmet Ali, 2001.

14. Change Institute, 2009 ; CERI, 1990.

15. Demetriou, 2003. 
transnationales. Il souligne aussi la préférence des élites pour le terme diaspora, plus déterritorialisé, plutôt que communauté, territorialisé.

Le présent article emploie le mot diaspora, sans se fixer sur son instrumentalisation, tout comme le mot grec paroikia, utilisé pour la communauté diasporique à Londres. Il peut se traduire par «établissement à l'étranger », «communauté en exil » ou « colonie ». Il emploie les deux termes, diaspora et communauté presque indistinctement, sans faire aucune tentative de typologie. Il en fait plusieurs usages pratiques, notamment pour faire allusion au mouvement (diasporisation), au sentiment d'appartenance à un pays référent ou au Royaume-Uni, mais il s'intéresse avant tout à la communauté reterritorialisée à Londres, la paroikia. Il cherche à saisir la manière dont des individus s'identifient, donnent sens au groupe, la façon qu'ils ont de construire et de mobiliser un sentiment d'appartenance à une terre lointaine. Tout cela, à travers leurs engagements politiques.

\section{Diaspora économique, paroikia politique}

La communauté diasporique chypriote des années 1920 jusqu'aux arrivées massives des années 1955-1965 - que j'étends ici jusqu'à 1974 - peut être qualifiée d'économique, c'est-à-dire motivée par la pénurie de travail à Chypre. Mais il faut souligner son caractère politique, pas tant pour inclure les acteurs de la gauche exilés de force ${ }^{16}$ que pour souligner son caractère politique per se: anti-impérialiste et anticoloniale, cette communauté mène farouchement la lutte des classes. C'est par cette lutte que les Chypriotes de la diaspora se sont engagés, pour beaucoup à Chypre d'abord, puis dans la métropole britannique, contre l'impérialisme britannique et contre tout autre impérialisme. Pour certains, la lutte a commencé à Chypre et s'est poursuivie dans la métropole par l'adhésion au parti communiste et une participation active au mouvement ouvrier. Pour beaucoup des arrivants des années 1950, l'engagement dans la lutte ouvrière contribue à leur intégration dans la diaspora. La référence à la disparité de classes sociales émerge alors, notamment au travers du rôle que les intellectuels assument aux côtés des ouvriers, depuis le recrutement et jusqu'à l'éducation des nouveaux venus.

C'est par leurs engagements politiques, notamment par la lutte des classes, que les Chypriotes intègrent la société britannique et, par ces engagements, leur démarche est tout autant transnationale qu'internationaliste. Des membres du parti communiste chypriote exilés à Londres produisent une réflexion et poursuivent la lutte anti-impérialiste sur le front britannique, mais aussi au plan international: ainsi, certains s'engagent dans la lutte armée contre le franquisme.

16. Demetriou, 2003. 
Leurs analyses notamment de la situation à Chypre sont en décalage critique face à la position prise par le parti communiste britannique sur les révoltes chypriotes de 1931. Celui-ci aligne en partie son analyse sur celle de son gouvernement et sur la doctrine marxiste-léniniste de l'auto-détermination nationale, alors que c'est la pauvreté qui motive les événements et la lutte des classes ${ }^{17}$. Comme l'observe Oackley (1979), les Chypriotes, influencés par Chypre, sont bien plus à gauche que ne l'est la scène politique au Royaume-Uni. Beaucoup rapportent même leurs fréquentes confrontations à l'hostilité de l'extrême droite anti-immigrants, et ce tout au long des années 1950-1960.

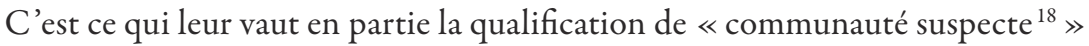
dès 1920 et jusqu'aux années 1960 : perçue par les autorités comme subversive, notamment en raison de l'adhésion importante de ses membres au parti communiste britannique, et comme criminelle, en raison d'un certain nombre de délits, elle est étroitement surveillée dans la métropole. Smith et Varnava renouvellent notre compréhension de la communauté chypriote de l'époque et notre vision de sa relation aux autorités, en montrant que cette surveillance s'étend à Chypre même, et entraine le contrôle et des restrictions au mouvement migratoire des sujets chypriotes. Les mesures réservées aux Chypriotes en 1935 sont celles appliquées en 1962 à l'ensemble des Chypriotes de tous les pays du Commonwealth.

Le caractère de la paroikia se construit sur cette identité politique qui fait de la lutte des classes une question centrale. Elle est le résultat des luttes et de jeux de pouvoir internes à la diaspora chypriote, notamment avec la formation de la Cypriot Brotherhood, composée de la droite chrétienne, pro-Enosis ${ }^{19}$, c'est-à-dire pro-union avec la Grèce, ou loyaliste britannique, fortement soutenue par les Britanniques pour agir contre le communisme et l'Enosis ${ }^{20}$.

\section{Diaspora en temps de conflit(s)}

Mais jusqu'à quand la diaspora est-elle économique, et la paroikia politiquement à gauche? Gilles Bertrand associe l'émigration chypriote à Londres au conflit. La violence, la surveillance et le contrôle associées à la lutte armée de l'EOKA ${ }^{21}$

17. Smith \& VARnaVa, 2000.

18. Ibid.

19. L'Enosis est l'Union à la Grèce.

20. Ibid.

21. BERTRAND, 2000. L'EOKA est le groupe paramilitaire chypriote grec qui s'est battu pour le retrait des Britanniques de Chypre et pour l'Enosis. 
(Ethniki Organosis Kyprion Agoniston) entre 1955-1960 pour le retrait britannique de Chypre et pour l'Enosis de l'île à la Grèce poussent de nombreuses personnes à émigrer vers la métropole de l'Empire britannique. Pourtant, une analyse plus générale des flux migratoires chypriote établit que l'émigration massive précède 1955, date du début de la lutte coloniale ${ }^{22}$ et qu'elle est liée aux phénomènes économiques. L'augmentation des flux d'émigration chypriote vers le Royaume-Uni observés entre 1958 et 1960 ne représente qu'une modification du lieu de destination de ces flux.

Certes, la violence n'est pas à exclure, mais est-elle le principal facteur d'émigration? Dans les années 1960, c'est beaucoup moins le(s) conflit(s) qui traverse(nt) Chypre qui amène(nt) à émigrer que les revers d'un demi-siècle de réformes britanniques, et notamment leurs effets négatifs au niveau écologique et économique ${ }^{23}$. Le contexte politique vient s'ajouter à la situation économique et conforter la décision de partir. La situation à partir des années 1960 est assez différente. La constitution de la République de Chypre en 1960 amène des centaines des Chypriotes turcs à quitter Chypre en même temps que les forces coloniales britanniques. Ils rejoignent Londres par allégeance à la Couronne qui leur a accordé des avantages pour leur réinstallation ${ }^{24}$.

Le retrait de la communauté chypriote turque de la République de Chypre et l'établissement des autorités séparées dans les enclaves en 1963 met fin aux aides au développement pour les Chypriotes turcs et détériore leurs conditions économiques. Les violences intercommunautaires contribuent à leurs déplacements massifs entre 1963 et 1967 vers les enclaves ou auprès de l'armée turque, et partiellement vers le Royaume-Uni, notamment après 1968, à la place du retour négocié ${ }^{25}$. La situation économique qui leur est infligée entre 1963 à 1967, la peur ou l'impossibilité du retour post-1968 contribuent à amplifier leur émigration ${ }^{26}$. Parmi les pistes offertes par les récits sur les déplacements chypriotes pour expliquer l'émigration chypriote grecque, on trouve le conflit entre nationalismes opposés, mais aussi des problèmes économiques. On peut comprendre que le conflit est une des raisons principales de déplacement, mais on peut très difficilement avancer que le conflit se déplace à Londres et qu'il

22. PÉCHOUX, 1977.

23. Karatsioli, 2009.

24. ØstergaArd-Nielsen, 2003.

25. En 1967, les deux autorités ont repris les négociations, et les autorités chypriotes turques ont encouragé la communauté à « retourner ».

26. Taylor (2015) évoque cette insécurité sans en faire une raison de déplacement, mais pour établir le lien avec la maison et le référent-origine. 
transforme le caractère de la paroikia chypriote. Notons d'ailleurs que ceux qui ont été forcés de se déplacer pour fuir la violence n'ont pas bénéficié du statut de réfugié qui aurait pu leur donner une légitimité. Durant ces années, une seule organisation a été créée pour soutenir à distance les revendications politiques des Chypriotes turcs dans le conflit. Mais rares sont les Chypriotes turcs qui se sont coupés de la communauté chypriote malgré le conflit. Ni la constitution de la République de Chypre en 1960 ni les déplacements économiques ou politiques dans les années 1960 n’ont réussi à transformer la diaspora.

\section{La diaspora, générée par le conflit: la frontière relocalisée}

C'est en 1974 que la nature de la diaspora s'est radicalement transformée. Après la fin de la guerre et la division de Chypre, alors que les Chypriotes grecs ont été déplacés de force vers le sud ${ }^{27}$, des Chypriotes turcs, avant même de décider de se réfugier en dehors du pays, quittent Londres pour Chypre. Ces quelques dizaines de Chypriotes turcs ne sont pas partis pour rejoindre leur village ou leur ville d'origine, mais pour retrouver leur communauté de village ou de ville installée dans la partie nord de l'île. Ils voulaient retrouver la sécurité de leur communauté à Chypre, mais peu envisageaient la construction d'un État; la présence massive de l'armée turque les a fortement influencés dans leur décision de retour. Ce déplacement n'a pas de valeur démographique et n'a pas transformé la nature de la diaspora. La même chose peut être dite pour les réfugiés chypriotes grecs qui arrivent à Londres après la guerre.

Parmi les 180000 personnes déracinées, près de 15000 ont été accueillies par la Grèce, et beaucoup ont trouvé un emploi au Moyen-Orient ou dans des pays socialistes ${ }^{28}$. Ni le Royaume-Uni, ni les autres pays de Commonwealth n'ont accueilli de Chypriotes grecs. Seules quelques centaines par an sont arrivées au Royaume-Uni au cours des trois années qui ont suivi la guerre ${ }^{29}$. Une chose est centrale: ce n'est pas une arrivée massive de réfugiés Chypriotes grecs à Londres qui a transformé la diaspora et qui a influencé le jeu des relations et des pouvoirs internes. C'est le fait de devenir réfugiés in absentia qui a été décisif. Et c'est le

27. Un petit rappel, les déplacés internes Chypriotes grecs n’ont pas forcément été bien accueillis : beaucoup de communautés leur ont refusé l'installation.

28. Péchoux, 1977.

29. KING \& BRIDAL, 1982, rapportent une baisse de la migration chypriote tout au long des années 1970 au Royaume-Uni; il n'y a eu qu'une très légère hausse entre 1974 et 1977: de 206 Chypriotes qui émigrent de Chypre en Grande-Bretagne en 1973 on en observe 649 en 1974, 529 en 1975, 726 en 1976, 781 en 1977 et puis une nouvelle baisse à 381 en 1978. 
déplacement de leur communauté, pas le leur qui a donné le droit au nom de «réfugiés ».

De «simple » diaspora économique, la diaspora chypriote s'est réinventée en 1974 en «diaspora générée par le conflit ${ }^{30}$ ». Générée par le conflit, mais surtout par la division, cette diaspora réinventée a généré elle aussi du conflit. Le conflit a partitionné l'espace diasporique chypriote alors qu'en même temps il devenait le principal moteur de son existence. Notons qu'en 1975 un tiers des Chypriotes vit à l'étranger ${ }^{31}$. Si auparavant la migration était une solution temporaire, devenus réfugiés in absentia, les Chypriotes doivent renoncer au retour ou à la réinstallation à Chypre ${ }^{32}$. Ils prennent conscience de leur situation de réfugié lorsqu'ils réalisent que leur séjour est forcément pérennisé.

Parler de diaspora générée (ou redéfinie) par le conflit oblige à prendre en compte sa transformation radicale et celle de son identité politique dès qu'elle devint porteuse de la cause chypriote grecque et de sa lutte pour la «justice ». S'opère alors une réorganisation interne accompagnée de bouleversements politiques, avec la rapide ascension de la droite nationaliste chypriote grecque, proche de la Brotherhood, laissant les communistes chypriotes mal à l'aise devant cette évolution.

Mais, soulignons-le, tous les Chypriotes grecs se mobilisent très vite, qu'ils soient de gauche ou de droite. Ils organisent des manifestations ${ }^{33}$, créent ou transforment l'identité des associations existantes de villages, dont certains désormais occupés par l'armée turque. Agir à Londres est extrêmement important : la Grande-Bretagne est aux côtés de la Grèce et de la Turquie, le troisième pays garant de la République de Chypre. Ils s'investissent très vite dans le lobbyisme à Londres et à l'international, pour devenir très rapidement le porte-parole du gouvernement de la République de Chypre ${ }^{34}$. Dans la diaspora, la lutte de classe ne disparaît pas : elle subit la même «défaite » que la gauche et devient secondaire face aux questions de guerre, déracinement et survie. D'une certaine manière, elle se patrimonialise, comme se patrimonialisent les relations entre Chypriotes grecs et Chypriotes turcs pour la majorité. Dans les années qui suivent la guerre, on observe la création des associations turques. Mais pour certaines, elles ne tendent pas vers la séparation avec la communauté chypriote grecque, elles contribuent

30. Lyons, 2006.

31. PÉCHOUX, 1977.

32. TAYLOR, 2015.

33. Constantinides, 1984.

34. Bertrand, 2000 ; Demetriou, 2003. 
activement à la perpétuation de l'intercommunautaire par la mise en place des associations comme Cyprus Community Centre (étude de cas: Safiye).

\section{La frontière déborde dans la ville}

Le conflit à Chypre a érigé une frontière à l'intérieur de la diaspora. L'absence de frontière territoriale devint vite problématique. La blessure de la frontière à Chypre cherchait sa réplique dans la construction de frontières dans la nouvelle ville. Malgré leurs efforts de ségrégation territoriale, en dépit de leur politique d'évitement de l'autre dans leur quotidien comme dans leur propagande, de façon paradoxale, les deux communautés se trouvaient indissociablement liées par le conflit. La frontière avec les Chypriotes turcs n'étant pas marquée territorialement comme c'était le cas à Chypre, ce voisinage suscitait des problèmes, surtout pour les Chypriotes grecs qui se plaçaient en position de victimes. La frontière territoriale devait se construire. S'éloigner de Camden pour s'éloigner de l'ennemi s'est avéré vain, car les Chypriotes turcs se déplaçaient aussi vers le nord. Ces changements démographiques ont contribué à ce qu'Haringay prenne le dessus sur Camden, puis qu'Enfield devienne le centre de l'immigration chypriote. Ensuite, l'amélioration de la situation économique individuelle entraîna un mouvement d'installation vers des quartiers dépourvus d'ennemis, souvent dans l'idée d'y faire venir les siens - d'abord la famille proche, puis des amis, ou d'anciens co-villageois. Mais, en dépit de tous ces efforts, cette stratégie pour se retrouver dans des quartiers sans ennemi s'est révélée vaine.

$\mathrm{Si}$ progressivement l'évitement quotidien s'est banalisé pour beaucoup au marché, à l'école, dans le quartier, bref dans la vie de tous les jours, d'autres mécanismes ont agi. La frontière ne pouvait pas être marquée territorialement et l'autre ne pouvait pas disparaître. Le zèle de beaucoup s'est manifesté par la nécessité d'exclure l'autre: dans les cas extrêmes, des étudiants chypriotes grecs ont abandonné leurs études à l'université après des longues batailles contre l'université qui acceptait les étudiants chypriotes turcs ou des Turcs dont la communauté et le pays étaient responsables de l'occupation militaire de Chypre (étude de cas: Maria). La transformation des configurations relationnelles et l'évolution de l'espace urbain ont eu des influences réciproques.

De manière générale, dans leurs déplacements, on peut dire que les migrants ont apporté avec eux des tensions et des conflits. Dans un premier temps, la lutte des classes a été le moteur d'intégration dans la société britannique, la visée anti-impérialiste depuis le centre de l'Empire britannique étant centrale. Puis, le conflit entre Chypriotes grecs et turcs s'est délocalisé à Londres par l'effet qu'il a produit à Londres même. De nouvelles frontières se sont construites et d'anciennes ont muté, disparu ou se sont étendues avec les déplacements même 
des migrants ailleurs dans la ville. Ainsi, on peut dire que la frontière à Londres est de nature multiforme et flexible, contrairement à la frontière rigide de Chypre qui sépare physiquement les deux communautés. Elle est marquée par la mutation du conflit en d'autres formes de conflictualité, comme celle de l'antagonisme généré par le marché de l'immobilier. Enfin, il est central de se demander s'il y a une concordance entre les deux frontières et notamment comment celle de Londres affecte à son tour celle de Chypre. Si le jeune Français de père chypriote qui n'a jamais vécu avec des Chypriotes turcs s'est mobilisé pour agir sur la frontière à Chypre que font les Chypriotes grecs et turcs de Londres?

\section{De la frontière à Londres à la frontière à Chypre : les formes de l'activisme transnational}

Le jeune Français de père chypriote n'a pas cherché à organiser son action avec la famille ou des membres de la communauté chypriote grecque de son père à Chypre. Il n'est pas allé au village de son père, toujours occupé par l'armée turque, pour revendiquer la restitution de ses terres. Et il n'a pas tenté non plus d'obtenir l'aide d'organisations chypriotes grecques en France, tout comme il n'a pas agi en collaboration avec des jeunes issus de la diaspora en France ou à Londres - où il avait pourtant des connaissances. Sidéré par la division persistante de Nicosie, il a noué des liens avec de jeunes inconnus de la communauté ennemie pour réclamer depuis la partie de la ville occupée par l'armée turque le dépassement du conflit et la réunification que l'intégration dans l'espace européen aurait dû apporter. Pour lui, la réunification de Nicosie, dernière ville divisée d'Europe, s'imposait.

Plus généralement, depuis la réunification de Berlin et de l'Allemagne en 1989, la chute du Mur et la fin de la guerre froide, invoquer Nicosie comme la dernière ville divisée de l'Europe donne une valeur ajoutée aux revendications des uns et des autres. La candidature de la République de Chypre à l'Union européenne en 1990 s'est accompagnée par une reprise des négociations avec les autorités chypriotes turques, en même temps que des initiatives bicommunautaires se mettaient en place pour rétablir le contact et engager les deux communautés dans un processus de rapprochement ${ }^{35}$.

Les manifestations des Chypriotes turcs de 2002 ont transformé la conception de la frontière et de l'espace. Ayant mobilisé une large majorité, près de $80 \%$ de la population chypriote turque, elles ont représenté le moment où la voix chypriote

35. Les réunions ont d'abord eu lieu en dehors de Chypre puis progressivement, en fonction des autorisations accordées par les autorités, sur l'île dans la zone tampon. Papadakis, 2005 ; Karatsioli, 2014. 
turque a retenti au-delà de l'État-marionnette de la Turquie qu'elles dénonçaient pour sa corruption économico-politique. Le nouveau refus des autorités chypriotes turques d'entrer en négociation avec les Chypriotes grecs à un moment où la réunification sous-entendait l'adhésion de Chypre à l'Union européenne a mené les Chypriotes turcs « dans la rue ». Les Chypriotes turcs ne voulaient plus être retenus par la Ligne verte; ils réclamaient leur droit à faire partie intégrante d'une «solution » et à l'Union européenne.

Beaucoup sont ceux qui attendaient que la manifestation gagne l'autre partie de la capitale divisée de Chypre. C'est ce débordement que ce jeune homme voulait provoquer quand il a hissé le drapeau européen en 2007. Il réclamait la sortie de l'inertie où se trouve englué le processus d'unification, comme le contact, rétabli en 2004, quelques jours avant l'adhésion de la République de Chypre à l'Union. Ce long processus d'ébullition de la frontière a-t-il eu des effets sur la diaspora chypriote à Londres? La circulation au-delà de la Ligne verte en 2004, interpelle les Chypriotes de Londres: elle établit la possibilité d'interaction avec l'ennemi, tout comme à Londres, et autorise en même temps la circulation dans tout le territoire, mais pas le retour. Cette concordance a-t-elle des incidences sur l'action des Chypriotes de Londres?

Nous étudierons l'activisme politique transnational des trois transmigrants à travers les liens qu'ils forgent avec leur pays d'origine désormais partitionné, et cela, à différentes échelles (famille, ville, village, communauté, État). Comment faire le lien chez les sociétés migrantes entre le pays d'origine et le pays de résidence ${ }^{36}$ quand sa communauté ethnique, villageoise, de quartier est d'un côté de l'île et ses possessions matérielles et ses souvenirs de l'autre ? Et comment s'établit la connexion? Enfin, sur quels imaginaires de l'État, de la patrie et du territoire se construisent les actions?

\section{Maria, ou le (droit du) retour comme fracture entre « déplacés internes 》 et «réfugiés»}

Maria, Chypriote grecque est née à Londres dans l'immédiat après-guerre, c'està-dire au moment de la division de 1974; ses parents ont été dépossédés de leurs biens et sont arrivés à Londres «sans rien dans les poches ». Depuis son enfance, elle parle de l'injustice commise envers les Chypriotes grecs, envers ses parents et elle-même, qui n'a pu jouir de la vie de ce village près de Kerynia dont on lui parlait tant. Dans le restaurant de ses parents, tout plat servi aux clients britanniques doit être accompagné d'informations sur la situation à Chypre; elle doit être douceamère comme l'est sa vie, teintée selon elle de l'amertume du conflit. Toute la

36. BAsCH et al., 1994. 
souffrance et tout le chagrin que ses parents réfugiés ont incarné, elle les a transformés en rage et en détermination à se battre contre l'injustice faite à son peuple. La réunification de l'île ne pourrait se faire que si, au préalable, justice était rendue aux Chypriotes grecs, victimes de la guerre. Les murs de son restaurant familial sont couverts de cartes de Chypre, de posters, tous les supports visuels qui pourraient informer ses clients de ses revendications. Les « 3 Rs» - Retrait des troupes turques de Chypre, Rapatriement de tous les colons Turcs, Retour pour tous les réfugiés - expriment sa vision et son engagement - entre autres - au sein de Lobby for Cyprus. C'est une position qu'observent certains partis à Chypre et qui s'aligne sur la position officielle de la République de Chypre ${ }^{37}$. À son grand regret et à celui d'autres Chypriotes grecs de l'organisation ou de la diaspora, cette ligne n'est pas suffisamment revendiquée par les Chypriotes grecs à Chypre.

C'est le cas de sa famille proche installée depuis la guerre à Limassol. Maria regrette de ne plus les voir lutter pour le droit de retour. Elle déplore de voir ses cousins urbanisés renoncer au retour au village, à la terre et à un mode de vie modeste pour préférer les richesses et les facilités de la ville. Cette attitude contraste grandement avec sa réalité londonienne. Vivre à Londres est un rappel constant de l'injustice qui a été faite à ses parents et à elle. Elle construit sa vie urbaine à partir d'un réseau de liens associatifs de village, mais pour sa famille chypriote qui se fond dans le tissu urbain, ces liens sont devenus formels. Et si Maria s'engage activement à travers l'association de son village et celle plus importante du Lobby for Cyprus, sa famille à Limassol reste passive; elle s'en remet aux mains de l'État mutilé. Pis encore, la possibilité de circulation entre les deux parties de l'île, rétablie en 2003, conduit sa famille à visiter le village ${ }^{38}$. Mais si la visite rendue à leur maison les a gênés sur le coup en raison des colons qui y habitent et de l'état pitoyable dans lequel elle était, ils ont apprécié et ont profité des prix bas dans cette partie de l'île. Ils n'ont pas réagi non plus face à la banalisation de la circulation qui permet aux Chypriotes turcs d'évoluer quotidiennement parmi eux.

Maria souligne une différence majeure entre les « déplacés internes » et les « réfugiés » à Londres, qui semble plus marquée lorsque la frontière devient tout aussi flexible à Chypre qu'elle est à Londres: la combativité de ces derniers. Ils assurent leur soutien actif et engagé à l'État dont ils prolongent la voix à Londres par leur lobbying. Ils construisent et pratiquent quotidiennement la frontière physique à Londres par l'évitement de l'ennemi, dans la rue, dans le quartier, à l'école et à l'université - Maria s'est opposée à l'inscription des Chypriotes turcs

37. Demetriou, 2003.

38. KaratSIOLI, 2014. 
et des Turcs à son université -, au point de quitter elle-même l'université. Elle a si fortement intégré la frontière et le confinement des Chypriotes turcs qu'elle refuse tout brassage. Elle veut éviter que Chypre ne devienne comme Londres; elle craint l'absence de solution et de justice pour les Chypriotes grecs. Elle veut transposer à Chypre la lutte préservée, vivace et centrale de Londres, car selon elle, elle a disparu de l'île, notamment en raison de l'enrichissement économique de la population et de la dissolution des liens du village d'origine dans la ville. Alors que les Chypriotes grecs à Chypre ont délégué l'action politique et la résolution du conflit à l'État, les Chypriotes grecs à Londres demeurent des acteurs de la frontière. Soucieuse de la situation - tout comme l'était Lobby for Cyprus -, incapable de mobiliser parmi ses liens familiaux à Chypre contre l'affaiblissement du «droit au retour», elle a décidé de s'engager en lobbying à Chypre même, d'abord à Limassol puis à Nicosie.

Dans son cas, les frontières entre le politique, l'économique et le culturel sont constamment brouillées: son restaurant, proposant une cuisine chypriote comme nationale, n'a pas le goût de la maison ("home"), mais bien celui de la maison, de la terre et de la patrie perdue ("home" et "homeland"). Le restaurant est un lieu construit sur la perte du territoire, la rupture dans le temps et dans le référent d'origine; de ce fait, il doit un jour - au moment de la «solution »disparaître. Dans sa démarche, l'attachement au village d'origine, "home", nourrit l'attachement au "homeland". Maria cherche à reproduire un espace dans lequel domine une conception pérennisée et inflexible du conflit. Pour elle, « traverser la Ligne » avant de gagner l'intégrité territoriale et la souveraineté de la République de Chypre sur tout le territoire est inconcevable. Mais, de manière paradoxale n'est-ce pas la Ligne imposée par la guerre et par l'ennemi que Maria est en train de consolider?

\section{Mustafa, ou construire l'État à travers les transferts économiques}

Mustafa avait environ 70 ans lorsque je l'ai rencontré en 2002. Politiquement actif dans la lutte armée chypriote turque pour le Taksim, la division de Chypre, paramilitaire à TMT (Türk Mukavemet Teşkilat ${ }^{39}$ ), arrivé à Londres vers la fin des années 1960, il a choisi d'y rester après la division de 1974. Et ce malgré son désir de s'installer dans cette partie du territoire où tous les Chypriotes turcs étaient enfin réunis sur un seul territoire, où les autorités chypriotes turques

39. TMT est l'organisation paramilitaire chypriote turque formée en 1958, pour se battre pour le Taksim, la division de Chypre en Chypre grecque et turque, la deuxième rattachée à la Turquie. Elle s'oppose à l'Enosis menée par EOKA qui a lutté pour l'union de Chypre (entière) à la Grèce. 
étaient souveraines et où l'armée turque, maintenant en force, assurait la sécurité. Ce n'était plus la même situation qu'en 1963 lorsqu'ils s'étaient retirés dans les enclaves, quand ils assuraient les opérations militaires eux-mêmes, disait-il. Et pourtant il n'est pas parti! Selon lui, c'était peut-être, en raison justement de cette expérience des années 1960, alors que les Chypriotes turcs avaient constitué leurs propres autorités pour gouverner les enclaves où ils s'étaient retirés, pour ensuite « retourner dans leurs maisons » en 1967 et 1968. Nul ne savait si cette division était une base de négociation pour une autre situation politique ou si c'était la situation même. Peut-être n'est-il pas parti en raison de sa famille - les enfants qu'il a eus entre temps - et/ou à cause de la supérette dont il était en train de devenir propriétaire à Londres. Quoi qu'il en fût, il a depuis assumé un rôle très actif depuis Londres, qui lui a progressivement donné une position privilégiée à Chypre associée à un mode de vie translocal, c'est-à-dire qui se mène sur deux lieux.

L'absence de soutien et de reconnaissance, les sanctions et les embargos imposés aux autorités chypriotes turques ainsi qu'à la «RTCN » établie en 1984 ont contribué à l'affaiblissement des moyens pour les Chypriotes turcs et à leur enclavement progressif. La dépendance des Chypriotes turcs - et des autorités à la Turquie pour leur subsistance et leur ravitaillement s'est vite décuplée. Les Chypriotes turcs de Londres se sont montrés actifs dans le contournement de ces sanctions en aidant les structures du « pays » et les Chypriotes turcs à subsister. Mustafa a consacré une part de ses profits à des transferts de fonds économiques plus ou moins réguliers vers ses sœurs et leurs familles. Dans les années 1990, il a hébergé ses neveux pendant leurs études et les a aidés à obtenir des papiers britanniques, en échange de leur travail dans sa supérette. Il a tout autant contribué à la construction de l'État, à travers des transferts économiques annuels en faveur de l'《État», et des transferts économiques «électoraux » au profit du parti dominant de Rauf Denktaş ${ }^{40}$, qu'il connaissait depuis les années 1950. En outre, il a réussi, en association avec d'autres entrepreneurs ${ }^{41}$, à contourner les embargos pour importer des produits chypriotes au Royaume-Uni. Par ailleurs, ces transferts lui ont valu les faveurs du régime, l'acquisition de considération et l'accumulation de richesses à Chypre Nord, tandis que les enfants de ses sœurs représentaient une main-d'œuvre pour sa supérette. Ainsi a-t-il pu partager sa vie chaque année

40. R. Denktaş est la figure de leader de la communauté chypriote turque qui a mené la communauté chypriote à la réalisation de l'objectif de la lutte armée, Taksim, la division. 41. L'autodéfinition comme "businessmen” de nombreux Chypriotes, propriétaires de fish and chips, de superettes ou autres petits commerces ajoute à leur prestige. 
entre huit mois à Londres et quatre mois à Chypre, où il profitait de sa maison construite en bord de mer.

À Londres, il entretient de bonnes relations avec des Chypriotes grecs dans le commerce, dans le voisinage, aux festivités. Il faut toujours distinguer entre la vie quotidienne et le projet politique, dit-il. Et de toute façon, ils sont à Londres. L'essentiel est dans la séparation à Chypre. Il participe très peu ou de façon très discrète aux activités de lobbyisme des Chypriotes turcs et des Turcs. Mais, il est très actif dans la collecte de renseignements sur les activités chypriotes à Londres, qu'il «partage» (c'est son expression) avec le régime chypriote turc. C'est un vieil art, dit-il, qu'il a développé dans son village natal dans les années 1950 et qu'il ne saurait abandonner tant que l'État chypriote n'est pas reconnu. C'est la construction de l'État chypriote turc qui prime pour Mustafa. Et elle doit se faire par tous les moyens, y compris l'oppression de la partie de la communauté résistante, que ce soit à Chypre Nord ou en diaspora et ce, jusqu'à sa maturité nationale. Il est d'ailleurs content de savoir que les autorités chypriotes turques ont légué une partie des fonds économiques reçus par les Chypriotes turcs en diaspora pour la construction de lieux de mémoire à Chypre Nord, c'est-à-dire de monuments qui commémorent la violence faite aux Chypriotes turcs. Et ce, pour rappeler à la population de plus en plus mécontente des Chypriotes turcs que la réunification, pensée comme le «retour » à la République de Chypre, n'est pas la solution.

Le cas de Mustafa vient montrer que les connexions ne se font pas uniquement sur des bases politiques, mais aussi par des transferts de fonds qui ont un rôle important, dans la construction du pays. Et, comme le soulignent Gardiner Barber et Lem (2012), les virements bancaires des migrants contribuent à la redistribution de ressources dans le monde, de manière multidirectionnelle et circulaire, mais aussi à solidifier les bases économiques d'un pays et à remédier à différents types d'embargo. Mustafa contribue non seulement à la construction d'un État, mais tout autant à son désenclavement, en réintégrant les Chypriotes turcs de l'« espace abject » ou du "no man's land" 42 dans l'économie globale dont ils sont «déconnectés ${ }^{43}$ ». Ce cas invite à revoir la question de la faiblesse du lobbying chypriote turc au Royaume-Uni telle que la note Østergaard-Nielsen (2003), et ce, à partir d'un cadre plus étendu de l'action politique. Mustafa menait un mode de vie translocal, fondé sur des liens familiaux, financiers et politiques qui bénéficient à la construction d'une entité politique, voire d'un nouvel État.

42. NAVARO-YASHIN, 2003.

43. Ferguson, 2002; Karatsioli, 2014. 


\section{Safiye, ou les cercles concentriques de l'appartenance communautaire}

Pour Safiye, l'attachement à Chypre est tout aussi inextricable qu'il est riche: une partie de sa famille proche, dont ses parents, vit à Londres depuis 1967 après s'être réfugiée dans une des enclaves chypriotes turques entre 1964 et 1967 ; une autre, du côté maternel, réside toujours dans le village mixte d'origine dans le « territoire sous contrôle de la République de Chypre »; une autre partie enfin a fui le village pendant la guerre avec les autres Chypriotes turcs du village pour « reconstituer » leur communauté sans les Chypriotes grecs dans un village de la partie nord de l'île. Par la dispersion territoriale de sa famille dans l'ensemble de Chypre et à Londres, Safiye a accès au village d'origine, à la communauté ancestrale chypriote turque installée dans la partie Nord, et à la communauté de cohabitation diasporique à Londres.

C'est dans cet espace diasporique qu'elle s'engage avant tout en poursuivant, dans la tradition de troisième génération de sa famille, un double engagement : en adhérant aux principes de gauche et dans l'action commune avec les Chypriotes grecs. Tout au long des années 2000, elle est active au sein de la gauche chypriote turque, notamment au travers de Turkish Cypriot Community Association fondé en 1976 et proche du parti de centre gauche chypriote turc CTP (Cumuburiyetçi Türk Partisi); elle participe à des campagnes politiques et elle œuvre, de manière plus formelle, à des événements organisés par AKEL (Anorthotiko Komma Ergazomenou Laou), le parti communiste chypriote (grec) au travers de Cyprus Community Centre, association créée en 1979 par des Chypriotes grecs et turcs. Ces affiliations soulignent son attachement à la communauté diasporique chypriote de gauche et son désir d'agir avec les membres de ces associations sur l'évolution du conflit à Chypre comme sur les conditions de la communauté à Londres - notamment par le suivi des papiers des Chypriotes turcs.

Au-delà de son engagement actif dans sa communauté chypriote londonienne de gauche, elle est aussi engagée dans les mouvements de la gauche britannique. Ce sont progressivement ces activités politiques qui deviennent prioritaires notamment lors de son passage au Parti socialiste des travailleurs (SWP) puis au Parti travailliste (Labor Party) et plus récemment encore par son soutien actif à Jeremy Corbyn dans Haringay, sa municipalité londonienne. Elle participe aussi à des manifestations anti-impérialistes et anti-guerres, dont celle engagée contre la venue de Henry Kissinger à Londres en 2002, Kissinger tenu pour être à l'origine du déclenchement de nombreux conflits un peu partout dans le monde et pour être responsable de la division de Chypre.

Elle met ses pas dans ceux de sa famille, mais en cherchant à tracer sa propre voie sur son lieu de résidence. Ses activités politiques sont essentiellement britanniques. 
La frontière à Chypre et ses transformations sont un point d'échange et parfois de convergence avec une partie de sa famille dont chaque membre mène sa propre action en fonction de son lieu de résidence. Elle est consciente que, tout comme la partie de sa famille restée au village ancestral, elle contribue par ses engagements à Londres à créer un avatar de la coexistence passée. Elle contribue à la continuation d'actions communes et à la reproduction d'un espace commun.

Pour terminer, tournons-nous vers Anna et Gül, toutes deux nées à Londres avant la guerre de 1974, également immergées depuis l'enfance dans l'espace politique de la gauche chypriote londonienne: elles décident indépendamment l'une de l'autre de s'installer à Chypre en 2007. C'est à Chypre qu'elles transforment leur engagement politique de Londres, engagement qu'elles qualifient «d'actions communes», par la construction de relations. Elles s'engagent aux cotés des femmes et hommes issus des diasporas britannique, états-unienne, sud-africaine, mais aussi australienne dans des activités de rapprochement qui se font jour à Chypre depuis les années 1990. «Être sur la frontière » telle est leur habitude, car c'est là que les choses bougent, mais contrairement à Safiye, elles choisissent le retour «sur» la frontière. Leur objectif est de transformer la frontière à Chypre, de contribuer, par-delà les clivages de chaque communauté, à la réunification de façon concertée.

\section{Conclusion}

La volonté d'agir sur le conflit à Chypre ne s'est pas seulement exprimée et élaborée par le biais de la voie étatique, ou par celui des institutions ou encore des organisations. L'étude des cas ici proposés contribue à montrer comment des Chypriotes grecs et des Chypriotes turcs agissent : que ce soit Maria qui mène plus loin l'action politique de lobbying des organisations de la diaspora, Mustafa qui néglige les institutions chypriotes turques à Londres pour soutenir directement la construction de l'État devenu le référent politique d'origine ou, enfin, d'autres comme Safiye qui agissent avec l'appui des institutions de gauche de leurs communautés d'appartenance - chypriote turque et chypriote grecque - pour consolider l'espace de coexistence londonien et contrer les tenants de la division à Chypre.

Loin d'en faire des idéaux-types, cet article s'interroge sur la manière dont leurs actions puisent à l'expérience londonienne de la frontière. Nous revenons donc à la question de départ concernant l'incidence que peuvent avoir la localité spécifique de Londres ainsi que la coexistence avec l'ennemi sur les actions politiques transnationales des migrants chypriotes. Dans les activités politiques de Maria et de Safiye, le caractère politique de la paroikia chypriote est facilement 
discernable. Les actions de Maria concordent avec la lutte devenue hégémonique de la paroikia dans sa réclamation de justice pour les Chypriotes grecs. Tout comme celles de Safiye, ses actions s'inscrivent dans un espace politique de gauche qui ne fait plus l'identité dominante de la diaspora, tout en reproduisant un espace politique et social de coexistence sur une position politique de gauche désormais périphérique. Quant aux activités politiques de Mustafa, elles tournent le dos à la paroikia comme espace politique, mais pas comme espace social.

À creuser davantage dans l'imaginaire de ces acteurs individuels, dans leur manière de comprendre et de classifier le monde, tout comme dans leur façon d'envisager le proche et le lointain ${ }^{44}$, on voit combien les pratiques londoniennes locales s'étendent pour englober la frontière chypriote dans la recomposition de la frontière. En d'autres termes, l'«imaginaire fondé sur le lieu » (place-based imagination) qui motive l'action de ces individus apparaît comme translocal. Pour mieux saisir cette connexion translocale de la frontière, nous avons suivi les trois acteurs dans leur production d'un espace social transnational ${ }^{45}$, à laquelle les migrants participent dans le quotidien et dans le processus politique de leur pays d'origine, tout en constituant une main-d'œuvre pour le pays d'accueil.

Les activités de Maria et Mustafa offrent deux expériences contrastées de cet espace. La disparité entre les politiques de la diaspora, actées par Maria, et celles de leur pays d'origine ont un effet négatif sur son espace social transnational lorsqu'elle tente en vain d'influencer les politiques à l'étranger et lorsqu'elle cherche à radicaliser les politiques dans le pays d'origine. Plus elle tente de matérialiser son imaginaire politique à Chypre, plus elle s'écarte d'un espace positif. L'activisme politique, la combativité de la diaspora comme extension de l'État, le droit de retour comme actuel retour, la conservation des liens de village par des associations, tout cela provoque une fracture très importante entre ceux qui sont devenus réfugiés par un déplacement à l'intérieur de Chypre et ceux devenus réfugiés par un déplacement à l'étranger (ici, à Londres). Elle considère agir sur la frontière à Chypre lorsque celle-ci devient flexible et par là même impose son contrôle (tout comme à Londres), mais aussi quand l'État perd le contrôle sur l'avenir du pays par la frontière: la circulation entre les deux parties rétablies par l'État illégal subvertit la souveraineté de la République de Chypre sur le territoire et sur ses citoyens. Il donne le signal de l'intervention des acteurs de la diaspora pour la consolidation de la frontière et par là, du droit au retour et à la solution.

Des activités politiques comme celles de Mustafa ont un impact positif sur leur espace social transnational. Le cas de Mustafa nous donne à voir un

44. DirLik, 2001.

45. Glick, Schiller \& Fouron, 1999. 
imaginaire fondé sur une pratique translocale, rapprochant d'emblée le lointain, «nouveau» référent originel. La production de cette translocalité vise à confirmer l'attachement à l'État et à s'assurer de son intégrité territoriale ainsi que de sa souveraineté. La production des translocalités n'affaiblit pas l'attachement à l'État ${ }^{46}$, elle le confirme et contribue même à donner à l'État non encore reconnu comme à sa population les moyens d'exister. Son imaginaire politique entre en jeu pour construire une relation positive entre la territorialité et la production de la translocalité. Mustafa ne va pas agir à un moment politique, à une phase du conflit plus qu'à une autre. Le conflit n'a pas seulement redessiné le tissu relationnel, il a divisé jusqu’à Nicosie, la capitale de Chypre.

Le cas de Safiye souligne le fait que la diaspora n'est pas seulement générée par le conflit ou le fait qu'elle génère du conflit. Des Chypriotes grecs et des Chypriotes turcs à gauche continuent de se politiser et de se socialiser. Cette socialisation due à une politisation à gauche reproduit les pratiques et les modes de vie de sa famille tels qu'elle les connaît grâce à la première génération de la diaspora à Londres. Elle incorpore et pratique la cohabitation à Londres où elle agit politiquement en tant que Chypriote turque, Chypriote et Anglaise (elle n'accepte pas d'être appelée Britannique) en même temps qu'elle préserve un espace social transnational positif.

Leurs actions politiques transnationales diffèrent de celle du jeune homme issu de la France. L'imaginaire de ce jeune homme est construit sur la base des idéaux de liberté et de paix caractéristiques d'un jeune homme du centre de l'Europe. La religion érigée en catégorie d'appartenance et d'action est influencée tant par la manière dont elle émerge dans l'école grecque qu'il fréquente le samedi et plus généralement dans la communauté grécophone hellénique auxquelles participent les Chypriotes grecs en France. Elle est aussi influencée par la guerre de religions en France au XVI ${ }^{e}$ siècle et la coexistence du catholicisme et du protestantisme.

Cette vue sur la diaspora offre ainsi une compréhension renouvelée du conflit chypriote. Elle propose d'aller au-delà d'une approche qui voit la diaspora ou les migrants comme témoins de l'histoire, officielle ou subalterne ${ }^{47}$, ou d'une approche qui place les réfugiés dans la perpétuelle recherche de la maison (bome) et de la patrie (homeland) ${ }^{48}$. Elle s'intéresse à l'action des migrants sur l'état du conflit et de la frontière que ce soit individuellement ou collectivement. Elle ne fait pas l'étude des organisations, de l'instrumentalisation de la diaspora ou

46. Appadurai, 1996; Gupta \& Ferguson, 1992.

47. Canefe, 2002; Chatzipanagiotidou, 2012.

48. TAYLOR, 2015. 


\section{CAHIERS BALKANIQUES}

176 Politique et sociétés à Chypre aujourd'hui

de la constitution des diasporas en idéaux-types ${ }^{49}$. Elle stipule la nécessité de situer la diaspora dans l'espace transnational et de reconsidérer la relation entre territorialité, espace transnational et État-nation par-delà une perspective qui fait annuler l'État-nation par le transnationalisme.

\section{Bibliographie}

\section{Monographies}

Appadurai Arjun, 1996, Modernity at Large, Cultural Dimensions of Globalization, University of Minnesota Press, Minneapolis, 229 p.

Basch Linda, Glick-Schiller Nina \& Szanton-Blanc Christina, 1994, Nations unbound: Transnational Projects and the Deterritorialized Nation-State, Gordon \& Breach, New York, 356 p.

Hadjiyanni Tasoulla, 2002, The Making of a Refuge: Children Adopting Refugee Identity in Cyprus, Praeger Publishers, Westport, 264 p.

Change Institute, 2009, The Turkish and Turkish Cypriot Muslim Community in England: Understanding Muslim Ethnic Communities.

Karatsioli Barbara, 2009, Quand les ennemis habitent ensemble: dynamiques du conflit et de la paix dans les cohabitations entre Chypriotes grecs et turcs, thèse soutenue à l'EHESS, non publiée.

Loizos Peter, 2003, Grace in Exile, Moufflon Publications, Nicosia, 175 p.

Navaro-Yashin Yael, 2012, The Make-Believe Space: Affective Geography in a Post-War Polity, Duke University Press, Durham. 296 p.

Papadakis Yiannis, 2005, Echoes From The Dead Zone. Across The Cyprus Divide, I.B. Tauris, London, $224 \mathrm{p}$.

Smith Hazel \& Stares Paul (ed.), 2007, Diasporas in Conflict. Peace-makers or Peace-wreckers?, United Nations University Press.

49. Bertrand, 2000 ; Demetriou, 2003 ; Adamson \& Demetriou, 2007. 
TAYLOR Helen, 2015, Refugees and the meaning of Home: Cypriot Narratives of Loss, Longing and Daily Life in London, Palgrave MacMillan, London, 188 p.

Tocci Nathalie, 2004, EU Accession Dynamics and Conflict Resolution, Ashgate, London, $216 \mathrm{p}$.

\section{Articles et participation à des ouvrages}

Adamson Fiona \& Demetriou Madeline, 2007, "Remapping the Boundaries of 'State' and 'National Identity': Incorporating Diasporas into IR Theorizing”, European Journal of International Relations, vol. 13, nº 4, pp. 489-526.

Bertrand Gilles, 2000, « Chypre: Diaspora(s) et conflit », CEMOTI, vol. 30, $n^{\circ} 1$, p. 59-82.

Bhrolchárn Maire N., 1990, "The Ethnicity Question for the 1991 Census: Background and Issues", Ethnic and Racial Studies, vol. 13, n 4, pp. 542-567.

Canefe Nergis, 2002, "Markers of Turkish Cypriot History in the Diaspora: Power, visibility and identity", Rethinking History, vol. 6, n 1, pp. 57-76.

Ceri Peach, 1990, "The Muslim population of Great Britain", Ethnic and Racial Studies, vol. 13, n 3, pp. 414-419.

Constantinides Paul, 1984, "The Greek Cypriots: Factors in the Maintenance of Ethnic Identity" in Watson James (ed.), Between Two Cultures, Basil Blackwell, Oxford.

Chatzipanagiotidou Evropi, 2012, “The 'Leftovers' ofHistory: Reconsidering the 'Unofficial' History of the Left in Cyprus and the Cypriot Diaspora" in Bryant Rebecca \& Papadakis Yiannis (ed.), Cyprus and the Politics of Memory: History, Community and Conflict, I. B. Tauris, London, pp. 94-117.

Demetriou Madeleine, 2003, "Priming the Diaspora: Cyprus and the Overseas Greek Cypriots" in ØstergaARD-Nielsen Eva (ed.), International Migration and Sending Countries, Palgrave Macmillan, London. 
178 Politique et sociétés à Chypre aujourd'hui

Dirlik Arif, 2001, "Place-based Imagination: Globalism and the Politics of Place", in Prazniak Roxanne \& Dirlik Arif, Places and Politics in an Age of Globalization, Rowman \& Littlefield, Lanham, Maryland, pp. 15-51.

Ferguson James, 2002, “The Global Disconnect: Abjection and the Aftermath of Modernism", in Inda Jonathan Xavier \& Rosaldo Renato (ed.), The Anthropology of Globalization: A Reader, Blackwell, Malden, pp. 136-153.

KIng Russell \& BRIDAL Janet, 1982, “The Changing Distribution of Cypriots in London", Studi Emigrazione: International Journal of Migration Studies, vol. 19, n 65, pp. 93-121.

LAdBury Sarah, 1977, “The Turkish Cypriots: Ethnic relations in London and Cyprus", in Watson James (ed.), Between Two Cultures: Migrants and Minorities in Britain, Basil Blackwell, Oxford.

LASSALlE Didier, 2006, «Question ethnique et question religieuse dans le recensement de la population britannique de 2001: Polémiques et enseignements », Revue européenne des migrations internationales, vol. 22, $\mathrm{n}^{\circ} 1$, p. 133-152.

Loizos Peter, 2007, "Generations in forced migration: Towards greater clarity", Journal of Refugee Studies, vol. 20, n² 2, pp. 193-209.

Loizos Peter \& Kelly Tobias, 2010, “The refugee factor in two protracted conflicts: Cyprus and Palestine compared", in Chatty Dawn \& Finlayson Bill (eds.) Dispossession and Displacement: Forced Migration in the Middle East and North Africa, British Academy/Oxford University Press, Oxford.

Lyons Terrence, 2006, "Diaspora and homeland conflict", in Kahler Miles \& WALter Barbara (eds.), Territoriality and conflict in an era of globalisation, Cambridge University Press, Cambridge, pp. 111-129.

Menmet Ali A., 2001, “The Migration of Turkish Speaking Communities to the UK" in Turkish Speaking Communities and education. No delight, Fatal publications, $232 \mathrm{p}$. 
Navaro-Yashin Yael, 2007, "Make-believe papers, legal forms and the counterfeit: Affective interactions between documents and people in Britain and Cyprus", Anthropological Theory, vol. 7, pp. 79-98.

Navaro-Yashin Yael, 2003, "Life is dead here: sensing the political in no man's land”, Anthropological Theory, vol. 3, pp. 107-125.

OAKLeY Richard, 1979, "The Cypriot Migration to Britain", in Saifullah Khan (ed.), Minority Families in Britain, Palgrave Macmillan Press, London.

Østergaard-Nielsen Eva, 2003, "The democratic Deficit of Diaspora Politics: Turkish Cypriots in Britain and the Cyprus issue", Journal of Ethnic and Migration Studies vol. 29, $\mathrm{n}^{\circ}$ 4, pp. 683-700.

PéChoux Pierre-Yves, 1977, «L'émigration des Chypriotes », Tiers-Monde, vol. $18, n^{\circ} 69$, p. 163-167.

Smith Evan \& Varnava Andrekos, 2017, "Creating a 'Suspect Community': Monitoring and Controlling the Cypriot Community in Inter-War London", English Historical Review, vol. 132, pp. 1149-1181.

SERT Deniz Şenol, 2010, "Cyprus: Peace, return and property", Journal of Refugee Studies, vol. 23, n 2, pp. 238-259.

WALDINGER Roger, 2006, «"Transnationalisme" des migrants et présence du passé », Revue européenne des migrations internationales, vol. 22, n 2, p. 23-41.

Zetter Roger, 1991, "Labelling Refugees: Forming and Transforming a Bureaucratic Identity" in Journal of Refugee Studies, vol. 4, n 1, pp. 39-62.

ZetTer Roger, 2007, "More labels, fewer refugees: Remaking the refugee label in an era of globalization", Journal of Refugee Studies, vol. 20, n 2, pp. 172-192.

Résumé: cet article cherche à comprendre comment le conflit chypriote se délocalise et se transforme à Londres. Il s'interroge sur la manière dont la frontière recomposée et intangible agit sur la frontière physique à Chypre, et comment les deux frontières interagissent. En partant de l'intérêt porté à l'activisme 
transnational chypriote, l'article propose en premier lieu une vue de l'espace diasporique chypriote à travers les engagements politiques des migrants. Il montre que la frontière entre Chypriotes grecs et Chypriotes turcs ne devient pertinente qu'à la suite de la guerre et de la division à Chypre en 1974. La diaspora jusqu'alors déterminée par la lutte des classes est transformée par le conflit, principalement par les Chypriotes grecs, devenus réfugiés in absentia, la migration temporaire étant désormais permanente. Il propose ensuite de suivre les engagements transnationaux de trois Chypriotes de la diaspora dans le conflit - et la paix - afin de cerner les transformations qui se font jour au sein de cette diaspora, approche qui permettra de comprendre comment elles agissent - ou proposent d'agir - sur la frontière physique à Chypre.

Mots-clefs : diaspora, conflit, frontière, Chypriotes grecs, Chypriotes turcs, relations intercommunautaires, réfugiés, migrants, transnationalisme, Londres, Chypre, $\mathrm{XX}^{\mathrm{e}}$ et $\mathrm{XXI}^{\mathrm{e}}$ siècles, anthropologie politique

Abstract: This paper examines the transformation of the Cypriot conflict through its relocation in the London diaspora. It juxtaposes the newly recomposed and intangible border in the diaspora to the physical one in Cyprus, showing how the two connect and interact. It recalls the historical transformations of the London Cypriot diaspora to explain the appearance of the border between Greek and Turkish Cypriots after the 1974 war and division of Cyprus. It deconstructs the idea of a massive Greek Cypriot refugee influx to the UK and shows how Cypriot refugees who were forced to make a temporary stay permanent, with no prospect of return, transformed and reinvented the diaspora and its power relations. It explores those transformations through the analysis of the political transnational engagements of three Cypriots in the diaspora. This work then shows how this recomposed and intangible border connects with, interacts with and impacts the physical border in Cyprus.

Keywords: diaspora, conflict, border, Greek Cypriots, Turkish Cypriots, intercommunal relations, refugees, migrants, transnationalism, London, Cyprus, $20^{\text {th }}$ \& $21^{\text {st }}$ centuries, political anthropology

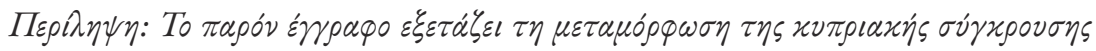

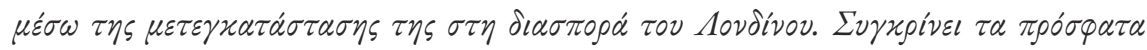

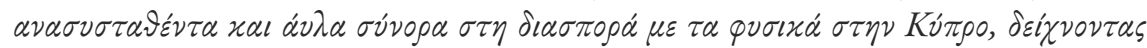

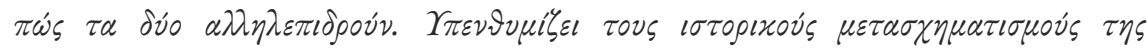

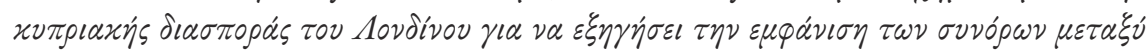

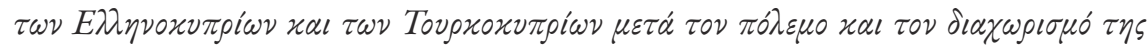

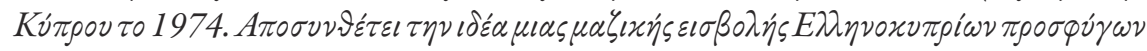

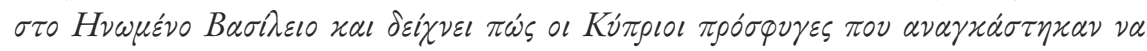




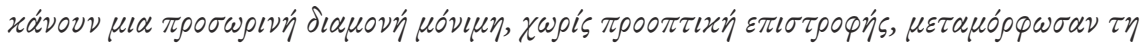

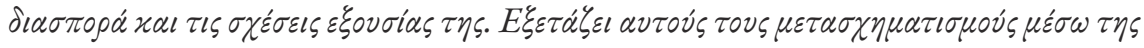

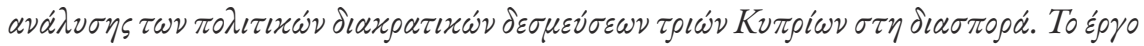

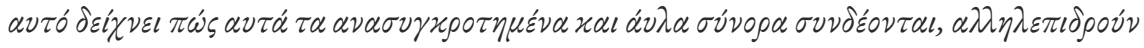

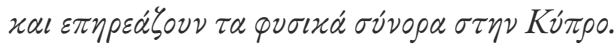

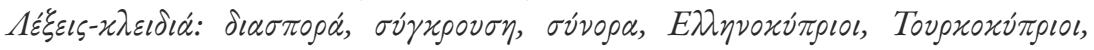

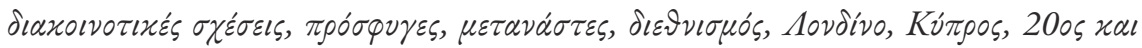

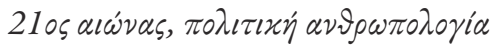

Anabtar kelimeler: diaspora, çatışma, sınır, Kıbrıslı Rumlar, Kıbrılı Türkler, topluluklar arası ilişkiler, mülteciler, göģmenler, ulusaşırlık, Londra, Kıbrıs, 20. ve 21. yüzyullar, siyasi antropoloji

Клучни зборови: дијаспора, конфликт, гранища, кипарски Грии, кипарски Турии, меѓуопштински односи, бегалии, мигранти, трансначионализам, Аондон, Кипар, 20 и 21 век, политичка антропологија 